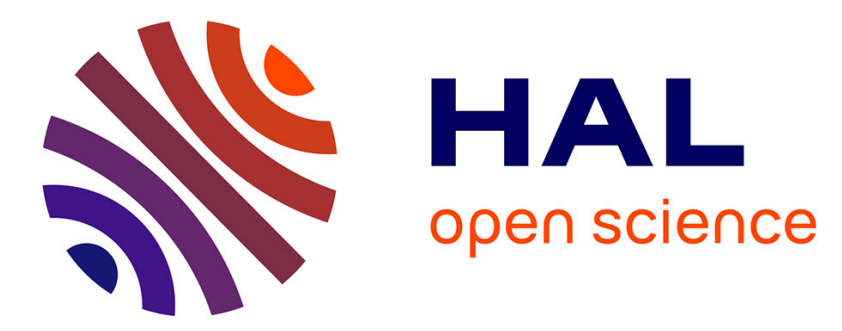

\title{
Concept and simulation of "Sys-Rééduc": Closed Muscular Chain Lower Limbs Rehabilitation Device
}

Lynda Seddiki, Kevin Guelton, Sebastien Leteneur, J. Zaytoon

\section{To cite this version:}

Lynda Seddiki, Kevin Guelton, Sebastien Leteneur, J. Zaytoon. Concept and simulation of "Sys-Rééduc": Closed Muscular Chain Lower Limbs Rehabilitation Device. 2007 IEEE 10th International Conference on Rehabilitation Robotics, Jun 2007, Noordwijk, Netherlands. 10.1109/ICORR.2007.4428478 . hal-01736804

\section{HAL Id: hal-01736804 \\ https://hal.univ-reims.fr/hal-01736804}

Submitted on 8 Jul 2020

HAL is a multi-disciplinary open access archive for the deposit and dissemination of scientific research documents, whether they are published or not. The documents may come from teaching and research institutions in France or abroad, or from public or private research centers.
L'archive ouverte pluridisciplinaire HAL, est destinée au dépôt et à la diffusion de documents scientifiques de niveau recherche, publiés ou non, émanant des établissements d'enseignement et de recherche français ou étrangers, des laboratoires publics ou privés. 


\title{
Concept and simulation of "Sys-Rééduc": Closed Muscular Chain Lower Limbs Rehabilitation Device
}

\author{
L. Seddiki, Student Member, IEEE, K. Guelton, Member, IEEE, S. Leteneur, J. Zaytoon
}

\begin{abstract}
This paper deals with a concept of a lower limbs closed loop rehabilitation device. The human force applied to this device is considered as an external disturbance to the system dynamics. A control structure was proposed to track desired trajectories and to attenuate the external disturbances. Stability conditions are given in terms of Linear Matrix Inequalities (LMI) using a quadratic Lyapunov function and an $\mathrm{H}$-infinity criterion. Finally, the simulation of the designed device on an isokinetic movement illustrates the efficiency of the proposed control structure.
\end{abstract}

\section{INTRODUCTION}

T ECHNICAL assistance to functional rehabilitation was the subject of many studies during last years, see e.g. [1][2] and references therein. Thus, a lot of rehabilitation devices were designed within specific wishes expressed by the clinicians. For instance, Lokomat is a device for the gait rehabilitation of patients with neuromuscular traumatisms [3]. Another example is an open muscular chain lower limbs rehabilitation device developed by the CReSTIC and named Multi-IS- [4][5]. The design of a new device is made starting from specific needs, expressed by clinicians, in rehabilitation. Thus, the concerned muscular complex and the various rehabilitation techniques have to be taking into account with special cares. In this study, we are interested in knee rehabilitation and consequently, to the global lower limb muscular complex.

Two rehabilitation techniques can be used: the first one is named open muscular chain (OMC) which is characterized by strengthening isolated muscular group whereas the second one, named closed muscular chain (CMC), is characterize by recruiting both the agonist and antagonists muscular groups that contributes to the movement. These two techniques are complementary and bring in various stages of rehabilitation protocols established by the clinicians.

This work was supported by the French Ministry of research and the "Région Champagne-Ardennes" within the framework of the CPER "Sys-Rééduc".

L. Seddiki, K. Guelton and J. Zaytoon are with the CReSTIC-URCA, Moulin de la House BP 1039, F-51687 Reims Cedex 2, France.

$\mathrm{S}$. Leteneur is with the rehabilitation center "La Rougeville", 89, av Henri Barbusse, F-5988 Saint-Saulve, France and with the LAMIH UMR CNRS 8530, University of Valenciennes, F-59313 Valenciennes Cedex 9 , France.

Corresponding author: Kevin Guelton, phone: +33-3-26-91-32-61; fax: +33-3-26-91-31-06; e-mail: kevin.guelton@univ-reims.fr
For technical reasons, it should be difficult to design a device that is able to reproduce simultaneously these two techniques. The only device that is able to reproduce both the techniques is named MotionMaker but remains to a very complex and expensive robotic structure that should only be used in the context of research studies [6]. OMC devices are commonly used in clinical context for the lower limbs rehabilitation. This is the case of isokinetic devices such as Cybex, Biodex or Multi-Iso [4][7]. More recently, CMC rehabilitation have been the subject of a particular attention because of its aptitude to stabilize the targeted joint while ensuring similar exercises used in the Daily life (gait, sit to stand, etc...). For instance, Moflex, Contrex LP or Erigo devices are CMC rehabilitation devices [8][9][10]. In this study, we propose the design of a new CMC lower limbs rehabilitation device named "Sys-Rééduc". This apparatus is designed to allow the rehabilitation of each lower limb independently. The goal of designing a new rehabilitation device is not to compete with the previously quoted devices. In fact, the objective is to mix their advantages and to bring some new specifications to allow a precise rehabilitation when soliciting the 6 degrees of freedom of the knee joint [11].

The paper is structured as follows. First, we will give some advantages and drawbacks of both rehabilitation techniques in order to justify the choice of the designed structure. Then, the dynamical model and the control structure based on disturbance attenuation will be presented. Finally, simulation results will illustrate the tracking performances.

\section{II. “SYS-RÉÉDUC" DEVICE CONCEPT}

A. Definitions, advantages and drawbacks of $\mathrm{MC}$ and CMC rehabilitation techniques

The exercises in open muscular chain are defined by the contraction of the agonist muscular group that allows the movement of the considered segment. In this rehabilitation mode, the foot is considered "free", figure 1. This approach is efficient and allows a fast recovery when strengthening isolated muscular groups. However, $\mathrm{OMC}$ has a major drawback, it causes constraints localized on the anterior cruciate ligament and on the patellar tendon which can generate significant pains during an intensive use [12][13]. Moreover, this technique tends towards to joint imbalance due to asymmerical tension of the targeted joint complex 
Exercises in CMC are defined by a simultaneous contraction of both the agonist and antagonists muscles for a considered movement. In the most case, these exercises are possible when the foot is in contact with a support, figure 2. Thus, the whole muscles contribute to the joint's actuation. Consequently, the musculo-skeletal tension is done in a symmetrical way and contributes to increase the joint stability. Thus, patellar pains are reduced during CMC rehabilitation compare to the $\triangle \mathrm{MC}$ rehabilitation [14][15]. Note that the benefit of $\mathrm{CMC}$ rehabilitation can be obtained for a longer period.

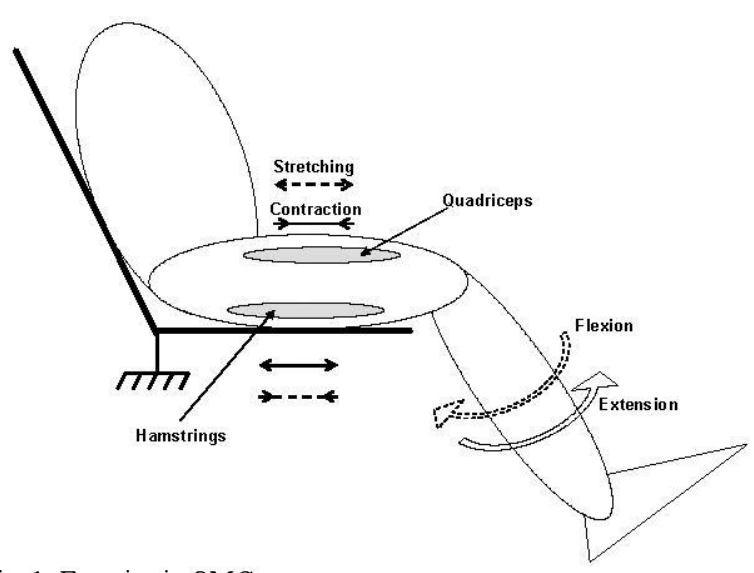

Fig. 1. Exercise in OMC.

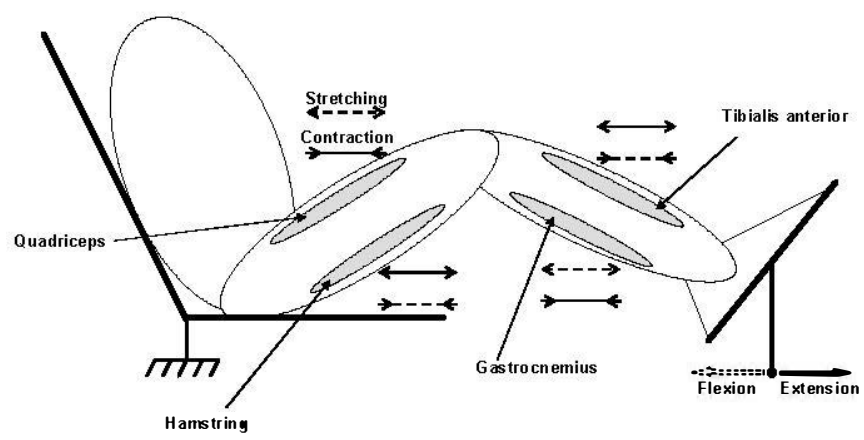

Fig. 2. Exercise in CMC.

Finally, $\triangle \mathrm{MC}$ and $\mathrm{CMC}$ rehabilitation techniques are complementary and make it possible to reach specific rehabilitation goals regarding to different pathologies. For instance, during long-term rehabilitation protocols, one can imagine to propose, in a first step, CMC exercises for stabilizing the whole joint complex and retaining from constraints generated by the $\mathrm{OMC}$. In a second step, when the patient's clinical state makes it possible, rehabilitation in -MC could be proposed for a specific muscular strengthening as the joint balance achieved in CMC is maintained.

\section{B. Kinematical concept of "Sys-Rééduc"}

At the CReSTIC, an isokinetic rehabilitation device, named Multi-Iso, is available [4]. In order to extend to a large panel of rehabilitation protocols, our objective is now to design an isokinetic CMC rehabilitation apparatus. By analogy with robotic systems, the mechanical complex \{lower limb, rehabilitation device\} must constitute a closed kinematical chain. For each lower limb, a closed kinematical chains, presented at figure 3 , is proposed and composed of the links $E_{1}, E_{2}, E_{3}, E_{4}$ and $E_{5}$ that are respectively the apparatus base, the thigh, the leg, the foot and the mobile foot's support. The joint between two links $i$ and $j$ is noted $L_{i, j}$

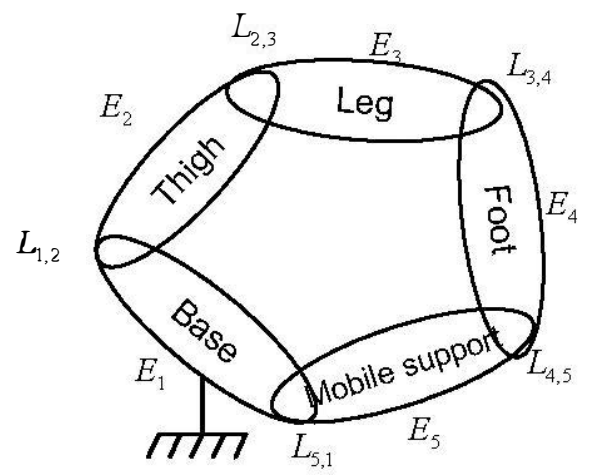

Fig. 3. Kinematic chain of a rehabilitation device of the lower limbs in CMC.

According to the kinematical chain depicted in figure 3 , the design of a new device remains in defining the nature of the joints $L_{1,2}, L_{2,3}, L_{3,4}, L_{4,5}$ and $L_{5,1}$. In that way, we assume that the human body consists in a set of rigid polyarticulated segments. In the sagittal plane, the whole kinematical structure, presented in figure 4 , is supposed to be composed of perfect joints such as:

- $L_{1,2}$ is the joint between the base and the thigh. The patient sits on a chair fixed to the base of the system. In that case, the trunk and the pelvis are also supposed to be fixed to the base. Then, $L_{1.2}$ is supposed to be a spherical joint corresponding to the coxo-femoral joint (hip).

- $L_{2,3}$ corresponds to the knee. This one is the main joint we wish to rehabilitate. Its complex kinematics was the subject of a previous study [9] and will not be detailed in this paper. In order to simplify the specification of the «Sys-Rééduc» kinematical concept, the knee will be represented as a hinge joint around the main lower limb flexion-extension axis.

- The joints $L_{3,4}$ and $L_{4,5}$ can be reduced to an unique joint $L_{3,5}$. To do that, a design constraint is to be made: the ankle axes and mobile support hinge joint, in the sagittal plane, are perfectly aligned with those of the foot mobile support. This constraint allows removing the foot from the kinematical chain. Then, $L_{3,5}$ consists of two hinge joints. The first one is the plantar flexion/dorsal flexion ( $L_{f d}$ ankle axis that is collinear with the main lower limb flexion-extension axis). The second one is the ankle internal-external rotation ( $L_{z e}$ ankle rotation in the tibia axis). Note that the adduction/abduction of the ankle was voluntarily omitted, insofar as it doesn't influence a lot the knee rehabilitation. 
- The joint $L_{5,1}$, between the foot's mobile support and the base is realised by a prismatic joint along the axis defined by the lower limb in complete extension.

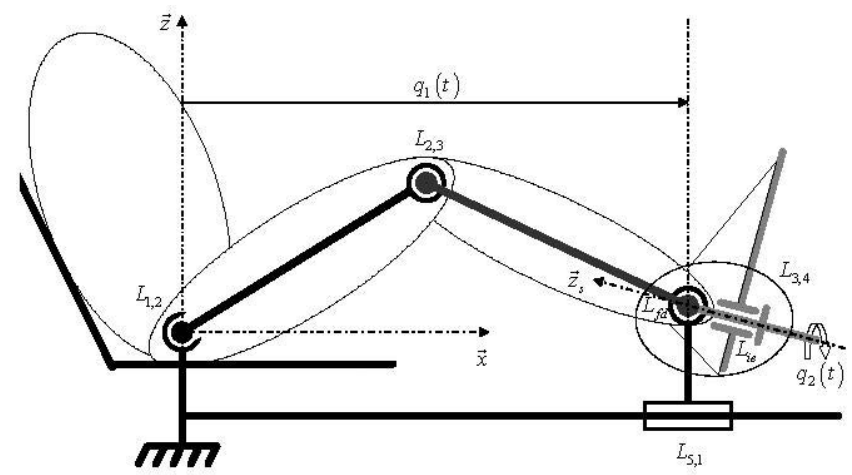

Fig. 4. Kinematic of Sys-Rééduc device in the sagittal plane.

Note that, according to the kinematics defined in figure 4, the internal-external ankle rotation $L_{i e}$ (along the $\vec{z}_{s}$ axis and depicted by the variable $\boldsymbol{q}_{2}(t)$ ) and the ranslation $L_{5,1}$ (along the $\vec{x}$ axis and located by the variable $\boldsymbol{q}_{1}(t)$ ) are sufficient to solicit the knee in its six degrees of freedom [11]. So these two axes will be motorized and the plantar flexion/dorsal flexion will remain passive (free or forced by a mechanical brake). In terms of rehabilitation, the motorization of the joint $L_{5,1}$ brings about a movement of the lower limb along the medial-lateral axis (flexionextension movement). This allows the rehabilitation of the flexor muscular complex and limits the constraints applied to the cruciate ligaments. Moreover, the foot's mobile support rotations allow the knee internal/external rotation and the plantar flexion/dorsal-flexion. These make possible a selective and precise muscular constraint. Then, rehabilitation or muscular strengthening can be practiced in a specific way during a desired movement. For instance, the lower limb movement with the foot in external rotation and in extension helps the stretching of medial hamstrings.

\section{MODELLING AND CONTROL OF «SYS-RÉÉDUC»}

\section{A. Generic control structure for rehabilitation devices}

A generic control structure for rehabilitation devices is presented in figure 5. This one was developed and experimentally validated in the case of $\mathbf{M C}$ rehabilitation on Multi-Iso [16] [20]. Thus, the proposed control structure of such a rehabilitation device is based on two levels depicted figure 5 .

- The continuous control level is devoted to stabilize the «Sys-Rééduc» device in closed-loop. To provide a safe behaviour, this level must ensure the stability of the mechanical part whatever the patient's effect to the machine is. In this case, we consider the human effect as an external disturbance $f_{p}(t)$ to be attenuated or to be rejected. In [16], the synthesis of a robust nonlinear control law with an $H_{\infty}$ criterion was proposed. Note that, this level does not allow the voluntary control of the system by the patient.

- A discrete level is devoted to provide the desired rajectory $y_{\alpha}(t)$ to be racked by the output $y(t)$ of the continuous dynamical system. This one is based on clinical specifications (isolinetic, isometric, isotonic ...). The principle remains on a discrete states machine [5][17] and allows the voluntary control of the system by the patient. Thus, according to the effort applied by the patient to the device $f_{p}(t)$, a desired and coherent rajectory $y_{\alpha}(t)$ is generated.

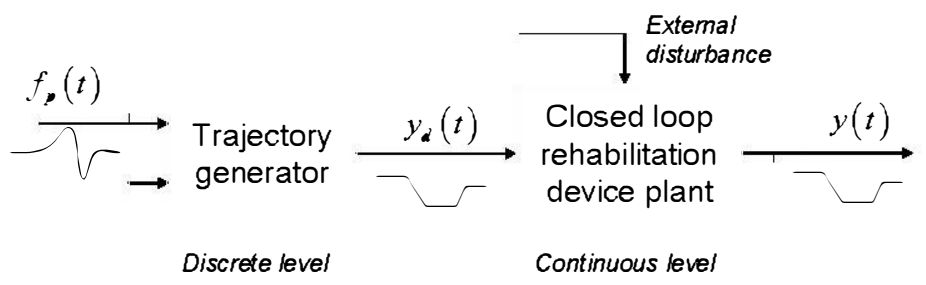

Fig. 5. Generic control structure for rehabilitation devices.

In the following, we will only consider the continuous level. Let us recall that various generic rehabilitation modes have been already translated in terms of discrete states machine [5][17] and will not be included in this article.

\section{B. "Sys-Rééduc" dynamical model}

The model is obtained through the Newton-Euler's equations. Figure 6 presents the mechanical scheme to be modeled. $C_{M 1}(t)$ and $C_{M 2}(t)$ are respectively the motors torques that allow the degrees of freedom movements associated with the variables $\boldsymbol{q}_{1}(t)$ and $\boldsymbol{q}_{2}(t)$. In order to synthesize the control law, we consider that the device is to be controlled via the motors torques while the patient applies external efforts (the force $f_{p}(t)$ along the $\vec{x}$ axis and the torque $C_{p}(t)$ around the $\vec{z}_{s}$ axis).

Note that the goal is now to validate in simulation the control structure proposed for the "Sys-Rééduc" concept and not to provide a fine modeling of a mechanical structure that is to be build (and not available at the moment). Then, in the following modeling, we neglect the frictions and backlash and only inertial masses will be tacking into account.

Moreover, using the control scheme depicted in figure 5, only the mechanical part less the human lower limb of the CMC kinematical structure have to be controlled as the patient effect to the device is considered as an external disturbances. In that case, the modeling remains to a robot with open chain mechanism. After isolating the foot's 
mobile support, the movement equations of the robot effectors (mobile support) are given by:

$$
\left\{\begin{array}{l}
\ddot{q}_{1}=\frac{C_{M 1}(t)}{a \cdot m}+\frac{f_{p}(t)}{m} \\
\ddot{q}_{2}=\frac{C_{M 2}(t)}{J}+\frac{C_{p}(t)}{J}
\end{array}\right.
$$

with $a$ the band wheel radius of the system realizing the translation along the $\vec{x}$ axis, $\boldsymbol{m}$ and $J$ the foot's mobile support mass and inertia.

Naturally, the model (1) can be rewritten as the following state space representation:

$\left\{\begin{array}{l}\dot{x}(t)=A x(t)+B u(t)+H \varphi(t) \\ y(t)=C x(t)\end{array}\right.$

where the vector $\varphi(t)=\left[\begin{array}{ll}f_{p}(t) & C_{p}(t)\end{array}\right]^{T}$ is considered as an external disturbance vector, $u(t)=\left[\begin{array}{lll}C_{M 1}(t) & C_{M 2}(t)\end{array}\right]^{T}$ is the input vector, $x(t)=\left[\begin{array}{llll}\dot{\boldsymbol{q}}_{1}(t) & \dot{\boldsymbol{q}}_{2}(t) & \mathbf{q}_{1}(t) & \mathbf{q}_{2}(t)\end{array}\right]^{T}$ is the state vector, $y(t)=\left[\begin{array}{ll}\mathbf{q}_{1}(t) & \mathbf{q}_{2}(t)\end{array}\right]^{T}$ is the output vector, $A=\left[\begin{array}{cc}0_{2 \times 2} & 0_{2 \times 2} \\ I_{2} & 0_{2 \times 2}\end{array}\right], \quad B=\left[\begin{array}{cccc}\frac{1}{a m} & 0 & 0 & 0 \\ 0 & \frac{1}{J} & 0 & 0\end{array}\right]^{T}$ and $H=\left[\begin{array}{cccc}\frac{1}{m} & \bullet & \bullet & 0 \\ \bullet & \frac{1}{J} & \bullet & 0\end{array}\right]^{T}$ are respectively the state matrix, the input matrix and the transfer matrix of the external disturbance, and $C=\left[\begin{array}{ll}\boldsymbol{\bullet}_{2 \times 2} & I_{2}\end{array}\right]$ the output marix.

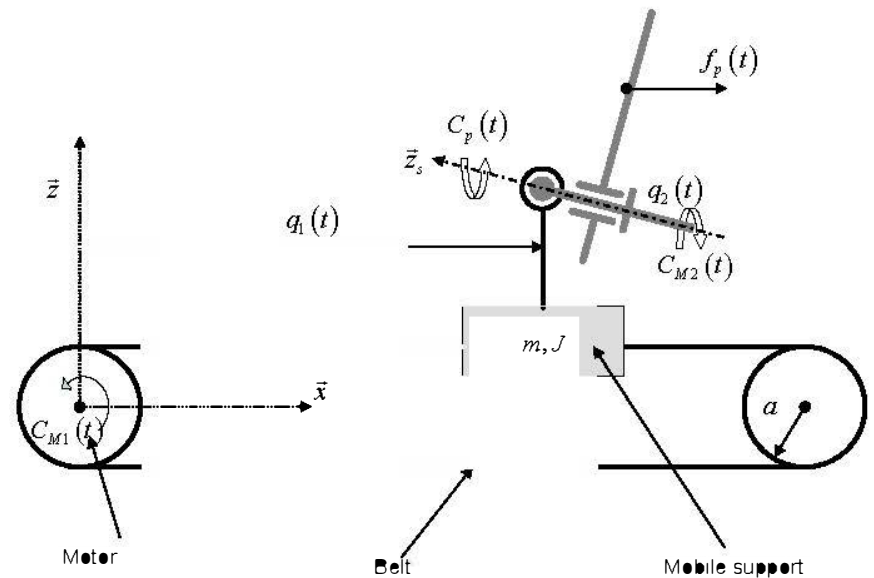

Fig. 6. Mechanical principle from the rehabilitation device.

\section{Control law design}

The objective is to guarantee the trajectory racking for the system described by (2). Then, the goal is to ensure the convergence of the racking error defined by $e(t)=x(t)-x_{\alpha}(t)$ with $x_{\alpha}(t)$ the state vector of desired rajectory.

We suppose that the state is available, the model is subject to external disturbances $\varphi(t)$ to be attenuated. In order to synthesize a control law that stabilizes the system subject to external disturbances, a robust control approach is needed. Thus, an $H_{\infty}$ criterion may be used since it allows attenuating external disturbances and ensures the security of the patient. This one is given by [18]:

$\int_{0}^{t f} e^{T}(t) Q e(t) d t \leq \eta^{2} \int_{0}^{t f}\left[\tau^{T}(t) \tau(t)+\varphi^{T}(t) \varphi(t)\right] d t$

with $Q>\bullet$ the weighting marix and $\eta$ the attenuation rate.

We consider the control law given by:

$u(t)=-K e(t)$

where $K \in \mathfrak{R}^{2 \times 4}$ is the feedback gain matrix.

The dynamic of the desired rajectory $x_{\alpha}(t)$ is given by the following state equation:

$\dot{x}_{d}(t)=A_{d} x_{d}(t)+\tau(t)$

with $A_{\alpha} \in \mathfrak{R}^{4 \times 4}$ an Hurwitz matrix and $\tau(t) \in \mathfrak{R}^{4}$ the input of the reference model.

Figure 7 shows the trajectory tracking control scheme with the reference model which will be used in the following

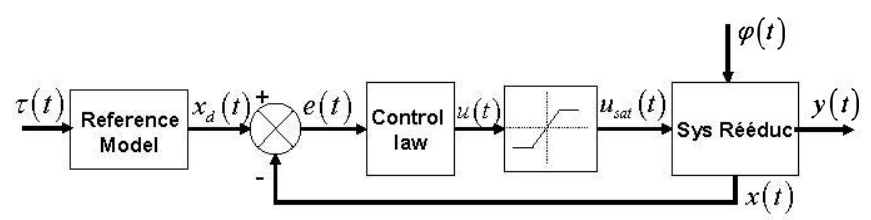

Fig. 7. Trajectory tracking control scheme with reference model.

Note that, for technical aspects related to the motors capability, the control signal is saturated.

The tracking error dynamic can be written as

$\dot{e}(t)=(A+B K) e(t)+\left(A-A_{\alpha}\right) x_{\alpha}(t)+H \varphi(t)-\tau(t)$ 
Let us consider $x_{e}(t)=\left[\begin{array}{ll}e(t) & x_{\alpha}(t)\end{array}\right]^{T} \in \mathfrak{R}^{\mathbf{2}}$ as an extended state vector. Its dynamic is given as:

$\dot{x}_{e}(t)=A_{e} x_{e}(t)+H_{e} \phi_{e}(t)$

with $A_{e}=\left[\begin{array}{cc}A+B K & A-A_{\alpha} \\ \boldsymbol{\bullet}_{4 \times 4} & A_{\alpha}\end{array}\right], \quad H_{e}=\left[\begin{array}{ccc}H & 0_{4 \times 2} & -I_{4 \times 4} \\ \boldsymbol{\bullet}_{4 \times 2} & 0_{4 \times 2} & I_{4 \times 4}\end{array}\right]$ and $\phi_{e}(t)=\left[\begin{array}{c}\varphi(t) \\ \boldsymbol{\bullet}_{2 \times 1} \\ \tau(t)\end{array}\right]$

In this case, the $H_{\infty}$ performance described by the inequality (3), can be rewritten according to the extended state $x_{e}(t)$ such as:

$\int_{0}^{t f}\left(x_{e}^{T}(t) Q_{e} x_{e}(t) d t\right) \leq \eta^{2} \int_{0}^{t f} \phi_{e}^{T}(t) \phi_{e}(t) d t$

where $Q_{e}=\left[\begin{array}{cc}Q & \boldsymbol{\bullet}_{4 \times 4} \\ \boldsymbol{\bullet}_{4 \times 4} & \boldsymbol{\bullet}_{4 \times 4}\end{array}\right]$

and $\phi_{e}^{T}(t) \phi_{e}(t)=\tau^{T}(t) \tau(t)+\varphi^{T}(t) \varphi(t)$.

The stability of (7) will be verified using the following Lyapunov quadratic function:

$v\left(x_{e}(t)\right)=x_{e}^{T}(t) \boldsymbol{P} x_{e}(t)$

with $\boldsymbol{P}=\boldsymbol{P}^{T}>\boldsymbol{\bullet}$ a $\mathfrak{R}^{\mathbf{8} \times \mathbf{s}}$ matrix

Indeed, the extended system described by (7) is stable along the rajectory $x_{e}(t)$ if $v\left(x_{e}(t)\right)$ decreases for $t \in \mathfrak{R}^{+}$within the $H_{\infty}$ performances (8), i.e. if there exist a marix $\boldsymbol{P}=\boldsymbol{P}^{T}>\boldsymbol{\bullet}$ satisfying the following constraint:

$\frac{d v\left(x_{e}(t)\right)}{d t}+x_{e}^{T}(t) Q_{e} x_{e}^{T}(t)-\eta^{2} \phi_{e}^{T}(t) \phi_{e}(t) \leq \bullet$

(10) can be developed in an extended form such as:

$$
\left[\begin{array}{l}
x_{e}^{T}(t) \\
\phi_{e}^{T}(t)
\end{array}\right]\left[\begin{array}{cc}
A_{e}^{T} \boldsymbol{P}+\boldsymbol{P} A_{e}+Q_{e} & \boldsymbol{P} H_{e} \\
H_{e}^{T} \boldsymbol{P} & -\eta^{2} I
\end{array}\right]\left[\begin{array}{l}
x_{e}(t) \\
\phi_{e}(t)
\end{array}\right] \leq \mathbf{0}
$$

(11) is obviously verified if:

$$
\left[\begin{array}{cc}
A_{e}^{T} \boldsymbol{P}+\boldsymbol{P} A_{e}+Q_{e} & \boldsymbol{P} H_{e} \\
H_{e}^{T} \boldsymbol{P} & -\eta^{2} I
\end{array}\right] \leq \boldsymbol{\bullet}
$$

Consider $Z=\boldsymbol{P}^{-1}>\boldsymbol{\bullet}$, after left and right multiplying (12) by $\left[\begin{array}{cc}Z & \boldsymbol{\bullet}_{\mathbf{z} \times \mathbf{8}} \\ \mathbf{0}_{\mathbf{8} \times \mathbf{8}} & I_{\mathbf{8} \times \mathbf{8}}\end{array}\right]$, it yields:

$\left[\begin{array}{cc}Z A_{e}^{T}+A_{e} Z+Z Q_{e} Z & H_{e} \\ H_{e}^{T} & -\eta^{2} I_{8 \times 8}\end{array}\right] \leq 0$

Consider $Z=\left[\begin{array}{cc}z_{1} & 0_{4 \times 4} \\ 0_{4 \times 4} & z_{2}\end{array}\right]$ and $\tilde{H}=\left[\begin{array}{ll}H & \boldsymbol{0}_{4 \times 2}\end{array}\right]$ with $z_{1}=z_{1}^{T}>$ and $z_{2}=z_{2}^{T}, \mathfrak{R}^{4 \times 4}$ matrixes. (13) can be developed with the marixes defined below (7) as follows:

$$
\begin{array}{cccc}
z_{1} A^{T}+A z_{1} & & & \\
+z_{1} K^{T} B^{T}+B K z_{1} & A z_{2}-A_{\alpha} z_{2} & \tilde{H} & -I_{4 \times 4} \\
+z_{1} Q_{1} & & & \\
z_{2} A^{T}-z_{2} A_{\alpha}^{T} & z_{2} A_{\alpha}^{T}+A_{\alpha} z_{2} & \boldsymbol{\bullet}_{4 \times 4} & I_{4 \times 4} \\
\tilde{H}^{T} & \boldsymbol{\bullet}_{4 \times 4} & -\eta^{2} I_{4 \times 4} & \boldsymbol{\bullet}_{4 \times 4} \\
-I_{4 \times 4} & I_{4 \times 4} & \boldsymbol{\bullet}_{4 \times 4} & -\eta^{2} I_{4 \times 4} .
\end{array}
$$

In (14), $z_{1}, z_{2}, K, A_{\alpha}$ and the attenuation rate $\eta$ are the variables to be find. The inequality (14) is obviously nonlinear within these variables. In this case, this cannot be solved using classical convex optimization tools such as the one describe in [19]. To obtain an Linear matrix inequality (LMI) formulation, some matrix ansformations are needed. Thus, one first apply the Schur complement on the first diagonal bloc of (14), it yields:

$$
\begin{aligned}
& \left(\begin{array}{l}
z_{1} A^{T}+A z_{1} \\
+z_{1} K^{T} B^{T} \\
+B K z_{1}
\end{array}\right.
\end{aligned}
$$

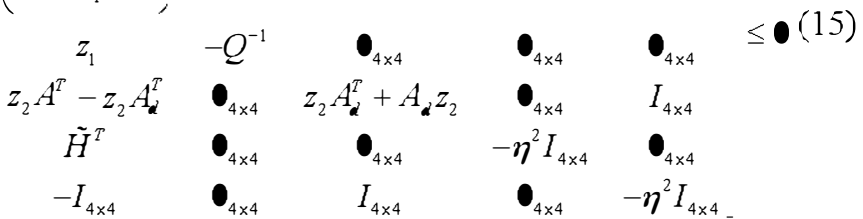

Afterwards, we use the bijective change of variables $M_{1}=K z_{1}, M_{2}=A_{\alpha} z_{2}$ and $\mu=\eta^{2}$. Then (15) becomes

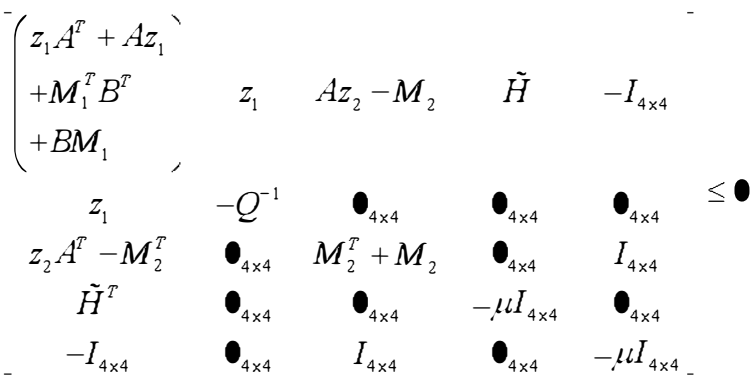


Finally, the control law design consist in solving the LMI (16). Thus, the gain matrix $K$, the Hurwitz matrix $A_{\alpha}$ and the attenuation rate $\mu=\eta^{2}$ are automatically obtained by means of the matrices $M_{1}, M_{2}, z_{1}$ and $z_{2}$ solving by LMI algorithms such as the Matlab LMI toolbox [19]. This ensures the trajectory racking closed loop stability with attenuation of the extemal disturbances

\section{Simulations Results}

The Trajectory tracking of the mechanical system (2) is simulated in closed loop via the control law (4). The model parameters used in this simulation are $a=\mathbf{0 . 0 2 5}(\mathbf{m})$, $\boldsymbol{m}=5(\mathrm{~kg})$ and $J=1\left(\mathrm{~kg} / \mathrm{m}^{2}\right)$. The design of the control law is obtained by solving the linear matrix inequality (16) with the Matlab LMI Toolbox [19]. Therefore, the performances was imposed by fixing the weighting matrix $\boldsymbol{Q}=\operatorname{diag}\left[\begin{array}{llll}\boldsymbol{0} .899 & \boldsymbol{0} .899 & \mathbf{0} 899 & \boldsymbol{0} .899\end{array}\right]$. Thus, the matrices and the attenuation rate leading to a feasible solution of (16) are given by:

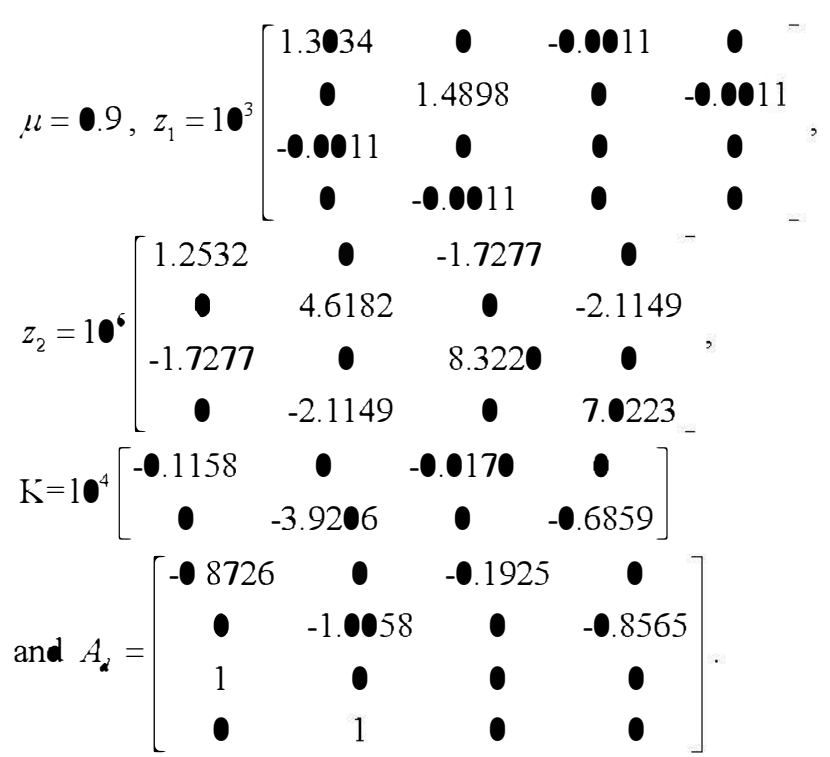

Note that the choice of the weighting marix $\boldsymbol{Q}$ is based on a compromise between the performances and the technical capabilities of the "Sys-Rééduc" device. Thus, the maximal motor load capacities are $\bar{C}_{M 1}=37.5 \mathrm{Nm}$ for $L_{5,1}$ and $\bar{C}_{M 2}=20 \mathrm{Nm}$ for $L_{i e}$.

One of the characteristics of the proposed LMI approach is that it allows obtaining automatically an Hurwitz reference matrix $A_{\alpha}$ whereas in the literature concerning rajectory tracking, its choice is difficult and often arbirary realized. In order to illustrate the disturbances attenuation, the simulation is realized by imposing external efforts that mimic the physiological constraint that is assumed to be maximal regarding to the effort that can be apply by a patient to "Sys-Rééduc". These are given by: $f_{\boldsymbol{p}}(t)=\frac{37.5}{a} \sin (6 t)$ and $C_{\boldsymbol{p}}(t)=20 \sin (5 t)$.

Note that the disturbance amplitudes correspond to the maximal motor loads and the frequency is quite slow $(\sim 1 \mathrm{~Hz})$.

In figure 8 , the simulated movement corresponds to an isokinetic extension of the lower limb with the velocity $\dot{\boldsymbol{q}}_{1}=0.8 \mathrm{~m} / \mathrm{s}$ realized between $t=1 \mathrm{~s}$ and $t=2 \mathrm{~s}$ from the initial position $\boldsymbol{q}_{1}(\mathbf{0})=\mathbf{0} .2 \mathrm{~m}$. This translational movement is combined to a sinusoidal internal-external rotation $\boldsymbol{q}_{2}=-(\pi / 4) \cos (2 t)$, presented in figure 9 .
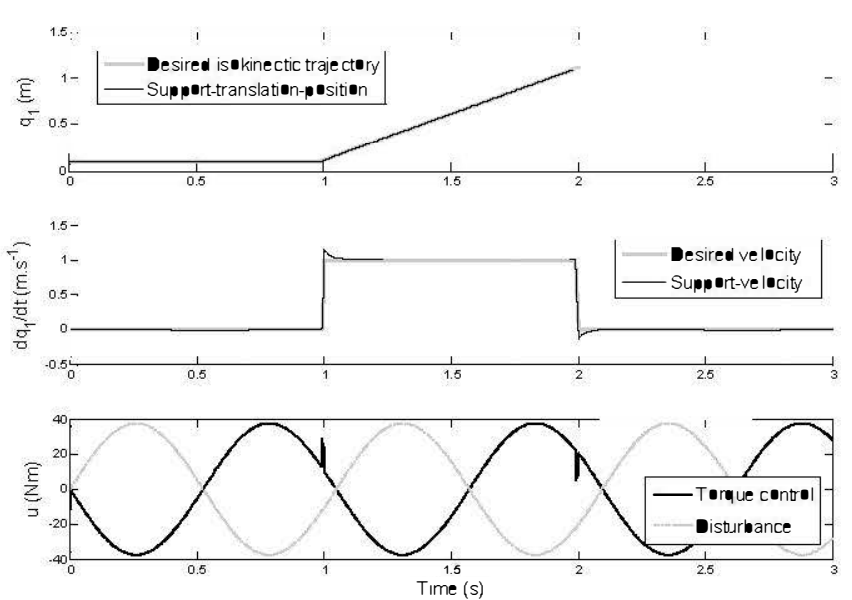

Fig. 8. Tracking trajectory of translation of the mobile support.
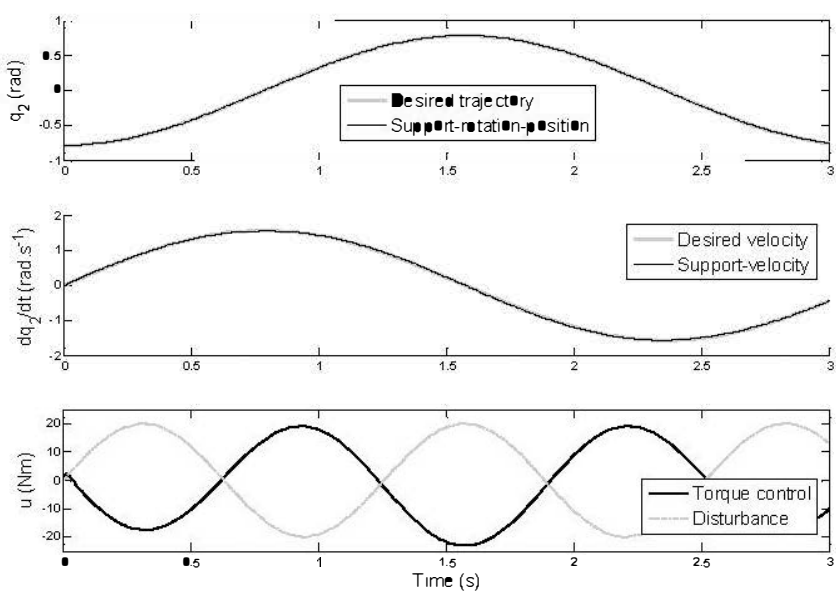

Fig. 9. Tracking trajectory for rotation of the mobile support.

Now, the following simulation, presented figure 10 for the lower limb isokinetic extension, will illustrate the performances of the proposed control law on the same movement but with "non physiological" extemal disturbances. That is to say a signal $f(t)$ that outperforms the effort that can be applied by a patient: 


$$
f(t)=500 \sin (6 t)+250 \sin (30 t)+125 \sin (60 t)+\operatorname{rand}(t)
$$

In this signal, the function $\mathrm{rand}(t)$ is used to simulate a noise measurement of the force applied by the patient. This one is a Gaussian disturbed signal with a variance of 1000 at a sample time of $0.01 s$
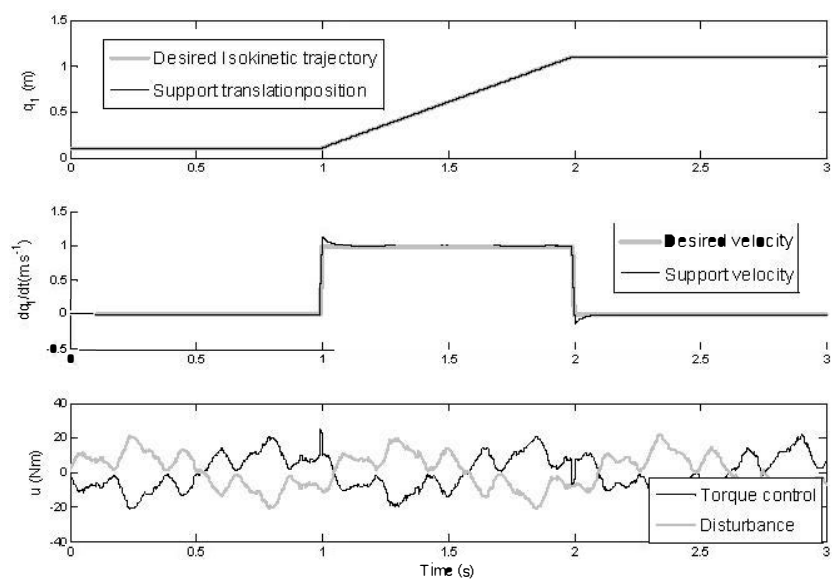

Fig. 9. Tracking trajectory for rotation of the mobile support.

These simulation results show that the $H_{\infty}$ control law successfully attenuates the external disturbances by means of compensations in the input signal.

\section{CONCLUSION}

In this paper, after having expressed the various advantages and drawbacks of the $\mathrm{OMC}$ and the $\mathrm{CMC}$ rehabilitation techniques, a new device concept for the lower limbs rehabilitation was proposed. This one allows, by means of a $\mathrm{CMC}$ rehabilitation, the six degrees of freedom solicitation of the knee joint. The control structure is based on two levels. The first one is a continuous level that ensures the stability of the mechanical system and the second level is based on a discrete state machine to generate the desired trajectories.

In this paper, the continuous level was studied. The efforts apply by the patient to the rehabilitation device were considered as external disturbances. Then, an $H_{\infty}$ control law has been designed in order to stabilize the system as well as attenuate the external disturbances. Stability conditions have been derived in terms of LMI. These one were verified with the considered system and simulation results have shown the efficiency of the proposed approach. Note that attenuating the external disturbances endows a safe behavior with the patient use of the rehabilitation device.

In a next paper, we will specify the discrete part according to many rehabilitation modes (isokinetic, isotonic, etc.). These trajectories will be parameterized in order to help the clinicians to specify an adapted rehabilitation protocol in reply to the considered pathology.

\section{ACKNOWLEDGEMENT}

The authors would like to thank Mrs. Maggy Bowl for their valuable advices relating to lower limb rehabilitation.

\section{REFERENCES}

[1] J.L. Dallaway, R. D. Jackson, P. H.A. Timmers, «Rehabilitation robotics in europe», IEEE Transaction on Rehabilitation Engineering, vol. $3 n^{\bullet} 1$, pp. $35-45,1995$.

[2] R. Riener, «control of robots for rehabilitation», EUROCON 2005, Belgrade, pp. 33-36, November, 2005.

[3] S. Jezernik, G. Colombo, M. Morari. "Automatic gait-pattern adaptation algorithms for rehabilitation with a 4-dof robotics orthosis». IEEE transations on robotics and automation, vol $2 \bullet, n \bullet 3$, pp 574-582, 2004

[4] S. Moughamir, "Conception et développement d'une machine de rééducation des membres inférieurs ». thèse de doctorat de l'université de Reims Champagne Ardenne, 1999.

[5] S. Moughamir, J. Zaytoon, N. Manamanni, L. Afilal. A system approach for control development of lower-limbs training machines»). Control Engineering Practice, pp. 287-299,10, 2002.

[6] P. Metrailler, V. Blanchard, I. Perrin, R. Brodard, R. Frischknecht, C. Schmitt, J. Fournier, M. Bouri, R. Clavel. «Improvement of rehabilitation possibilities with the MotionMaker ${ }^{\mathrm{TM}}$ ») IEEE International Conference on Biømedical Røbetics and Biemechatronics, pp 359-364, Pisa, Italy, 2006.

[7] «Les appareils d'isocinétisme en évaluation et en rééducation musculaire: intérêt et utilisation», Agence Natienal d'accréditation et d'évaluation en santé, fevrier 2001.

[8] C. Patterson, C. Raschner, H.P.Platzer, R. Puehringer. "A comparison of different tests to assess lower extremity left right strength imbalances»). IOS Press Isøkinetics \& Exercise Science, 14, 2, pp. 136$137,2006$.

[9] J. Petermann, P. Klein, A. Behrens, L. Gotzen, M. Schierl. « Efficacy of linear isocinetic versus dynamic auxotonic muscle training for the shoulder- A prospective, randomised, controlled study in men». IOS Isøkinetics \& Exercise Science, 10, pp. 39-40, 2002.

[10] G. Colombo, R. Schreier, A. Mayr, H. Plewa, R. Rupp. «Novel tilt table with integrated robotic stepping mechanism: Design principles and clinical application». IEEE, $g^{\text {th }}$ International Conference on Rehabilitation Røbøtics, pp 227-230, Chicago, USA, 2005.

[11]L. Seddiki, K. Guelton, L. Afilal, J. Zaytoon. « A 6 degrees of freedom kinematical model of the knee for the design of a new rehabilitation device». The $3^{\text {rd }}$ European Medical and Biological Engineering Conference. Prague, 2005.

[12] G.E. Lutz, R.A. Palmitier, K.N. An. « Comparaison of tibio-femoral joint forces during open kinetic chain and closed kinetic chain exercices 》, J. Bøne Jøint Surg, 75 A, pp. 732-739, 1993.

[13]EB. Bynum, R.L. Barrack, A.H. Alexaner. "Open versus closed chain kinetic exercices after anterior cruplate ligament reconstruction», A prospective randomized study. Am. J.Spørts Med, 23, 4, pp. 401-406, 1995.

[14]L. A. Steinkamp, MF. Dillingham, M.D. Markel. «Biomechanical consideration in patellofemoral joint rehabilitation), Am. J. Sperts Med, 21, 3, pp. 438-444, 1993.

[15] J.J. O'Connor, "Can muscle co-contraction protect knee ligaments after injury or repair». J. Bøne Jøint Surg, 758, 1, pp. 41-48, 1993.

[16] L. Seddiki, K.Guelton, B. Mansouri, JZaytoon. «H-infinity TakagiSugeno fuzzy control of a lower limbs rehabilitation device), IEEE, CCA, Munich, pp. 927-932, 2006.

[17] J. Zaytoon, S.Moughamir, N. Manamanni, L. Afilal, L. Angelloz. «Formal specifications of sequential control for training machines for the lower limbs», IEEE-EMBS, 4, pp. 3468-3471, 2001.

[18] K. Zhou, J.C. Doyle. «Essentials of robust control». Prentice Hall, Upper Saddle River, New Jersey, 1998.

[19] P. Gahinet, A. Nemirovski, A. Laub, M. Chilali, «LMI control Toolbox", The Mathwerks Inc, Natick, MA, 1995.

[20] L.Seddiki, K. Guelton, S. Moughamir, B. Mansouri, J. Zaytoon, «Modélisation T-S et commande $H_{\infty}$ d'une machine de rééducation des membres inférieurs), RS-JESA, 41, pp 199-218, 2007. 